\title{
Fibrosis-Induced Abnormalities of the Cardiac Conduction System and Malignant Arrhythmias in Patients with Chagas Disease \\ Centurión $\mathrm{OA}^{1 *}$ and García LB ${ }^{2}$
}

${ }^{1}$ Department of Health Sciences's Investigation, Sanatorio Metropolitano, Fernando de la Mora, Paraguay

${ }^{2}$ Hospital de Clínicas, Universidad Nacional de Asunción, San Lorenzo, Paraguay

Histopathological evidence of extensive fibrosis may produce myocardial areas where fibrous tissue separates the muscle fibers from each other. The dismally connected fibers in infected myocardial tissue with continuous fibro-degenerative modification may induce anomalous electrophysiological characteristics. Electrical coupling between adjacent fibers is difficult to occur when fibrosis surrounds groups of myocytes [1-4]. The microarchitecture and anisotropic characteristics may play an important role in re-entry by causing inhomogeneous and discontinuous propagation of the impulse. This non-uniform anisotropic property causes an irregular and fractionated propagation of the depolarization wave in the transverse direction [5-8]. Structural inhomogeneity or the common distinction in electrophysiological or ultra-structural properties plays a major role in the induction of re-entrant circuits and malignant arrhythmias due to the elevated probability of unidirectional block of the premature impulse and conduction delay [7-10].

The main arrhythmogenic substrates in Chagas Heart Disease (CHD) are necrotic and fibrotic lesions caused by inflammation of the myocardium. CHD is an illness closely associated to fibrotic and degenerative changes usually affecting the postero-inferior wall and apical region of the left ventricle, and the cardiac conduction system, namely, the sinus and atrioventricular nodes and the bundle branches. CHD is a parasitic zoonosis caused by Trypanosoma cruzi which is spreader by insects grouped in different species of Triatoma.

Transmission by blood transfusion, affected organs transplant, and oral transfers has also been explained [11,12]. Untreated Chagas' disease gets into a chronic phase after the primary infection that is initially asymptomatic or hidden. Approximately $35 \%$ of the patients infected with T. cruzi will provoke symptomatic heart disease years after the acute phase [13-15]. Generally, during the disease, the cardiac irregularities come out steadily around $20-30$ years following infection. Whereas, during the intense phase of the contagion, about $10 \%$ of the patients develop myocarditis that may result in serious form of CHD. When exposed to immunosuppression conditions, few patients having mild cardiac involvement, in chronic stage of this parasitic infection, may suffer from sudden exacerbation and acute heart failure. The physiopathology of this disease, and the comprehension of the scientific evidence currently available, demonstrates a complicated etiology. The mechanisms involved in the pathogenesis are also complex and multiple. The production of parasitic myocardial damage is evident $[16,17]$. An autoimmune phenomenal process was also explained $[18,19]$. Other demonstrated pathogenic mechanisms include microvascular alterations and autonomic denervation [20].

A year later of the discovery of Chagas disease, Carlos Chagas already noticed some abnormalities of the cardiac rhythm in few patients with chronic disease that were attributable to a diseased conduction system of the heart. Therefore, he strongly stated the participation of heart inclusion in patients with chronic Chagas disease [21]. For example, Carlos Chagas observed the occurrence of Premature Ventricular Contractions (PVC) as alteration of the cardiac rhythm. In the absence of electrocardiogram at that time as we know it today, in some cases of extreme bradycardia during physical examination he mentioned the presence of AV block as the direct cause. Carlos Chagas reported heart failure cases of patients and regular PVC who underwent autopsy studies. Many parasites along the interstitial mononuclear cell infiltration in the myocardium were found [22]. Hence, he stated that episodes of Sudden Cardiac Death (SCD) may occur during PVC for physical examination, as the cardiac form of the chronic disease [23].

Fibrotic lesions secondary to chronic cardiomyopathy in CHD can generate arrhythmogenic substrates that lead to the initiation and maintenance of ventricular arrhythmias. The deterioration of left ventricular systolic function was found to be an independent predictor of all-cause mortality in patients with chronic heart failure secondary to chronic CHD. In addition, ventricular tachycardia induced by exercise stress testing was found to be a predictor of SCD in patients with chronic CHD $[24,25]$. In an interesting nicely designed Magnetic Resonance Imaging (MRI) study [26], myocardial fibrosis was quantitatively investigated by MRI in patients with chronic CHD. It was demonstrated that myocardial fibrosis was present in $85 \%$ of patients with CHD, and that left ventricular ejection fraction was inversely correlated to myocardial fibrosis. It was very interesting to note that myocardial fibrosis was present in all patients with ventricular tachycardia. This finding suggests a role for fibrosis in the pathogenesis of malignant arrhythmias in patients with chronic CHD and also has a great clinical implication since ventricular arrhythmias associated with CHD have high incidence of morbidity and mortality [26].

Alterations of the cardiac conduction system such as atrioventricular and intraventricular conduction disorders are common manifestations of CHD and are usually related to left ventricular systolic dysfunction and ventricular arrhythmias. There is no specific or pathognomonic electrocardiographic feature of CHD. But, a detached right bundlebranch block or its connection with the left anterior hemiblock in patients with positive serology indicates chronic CHD [27,28]. CHD clinically often shows up as dilated cardiomyopathy having a tendency towards the development of apical aneurysms. It is a fibrotic disease that is normally situated in the posteroinferior and apical region of the left ventricle, the sinus node, and the conduction system below the bundle branch [27]. It has probable arrhythmogenic tendency that

*Corresponding author: Centurión OA, Professor, MD, PhD, FACC, FAHA Professor of Medicine, Asuncion National University, Department of Health Sciences's Investigation, Sanatorio Metropolitano, Teniente Ettiene 215 c/Ruta Mariscal Estigarribia, Fernando de la Mora, Paraguay, Tel: 595-021-421423; E-mail: osmarcenturion@hotmail.com

Received June 26, 2017; Accepted July 12, 2017; Published July 24, 2017

Citation: Centurión OA, García LB (2017) Fibrosis-Induced Abnormalities of the Cardiac Conduction System and Malignant Arrhythmias in Patients with Chagas Disease. J Bioequiv Availab 9: e79. doi: 10.4172/jbb.10000e79

Copyright: (c) 2017 Centurión OA, et al. This is an open-access article distributed under the terms of the Creative Commons Attribution License, which permits unrestricted use, distribution, and reproduction in any medium, provided the original author and source are credited. 
may lead to ventricular arrhythmias and SCD. Due to atrioventricular blockage and sinus node disease, the CHD is mostly linked with bradyarrhythmias. Patients with symptomatic CHD are at probable risk of SCD and hence it should be noted that SCD can be induced by CHD [29]. It was seen that few patients suffering from CHD, died unexpectedly during the when during chronic phase of the clinical evolution. Unpredictable SCD can be the main cause of mortality in patients suffering from Chagas disease. The occurrence of SCD can be found in number of subjects showing no previous symptoms or relevant symptoms during clinical follow-up [29]. There are some non-invasive auxiliary diagnostic methods that have some relative value as indicators of certain risk of developing arrhythmic events. The QRS duration is directly related to the left ventricular dilatation and inversely related to the systolic function. The duration of the filtered QRS measured by signal-averaged ECG is an independent predictor of SCD in CHD [28]. Preceding sudden and global asynchrony has been explained by the occurrence of repolarization disorders. The dispersion of the QT interval is present in $20-30 \%$ of infected subjects not showing any other disease symptoms. Hence, it is important to examine the dispersion of the QT interval and variability of the heart rate in Chagas disease as its connection with ventricular arrhythmia and sudden unpredictable death in patients infected with $T$. cruzi has been proved [17-19]. In this context, even subtle and nonspecific symptoms such as weakness or giddiness have much more importance as they may be expected of an episode of syncope. Patients with CHD mostly suffer from premature ventricular contractions and tachycardias, commonly connected with myocardial damage that produces re-entry phenomena. Constant ventricular tachyarrhythmias, in patients with CHD, do not originate from the apical aneurysm of the left ventricle but from the inferolateral part [30]. Usually, ablative procedures for ventricular tachycardia associated to CHD have poor outcomes. Since these reentrant ventricular rhythms forms within a progressive abnormality with several arrhythmogenic foci, radiofrequency ablation is not so active and hence cannot be contemplated as first preferred therapeutic approach. Though specific studies in chronic CHD are not available, the implantation of an Implantable Cardioverter Defibrillator (ICD) is advised to reduce the risk of sudden death in cases of clinical sustained ventricular tachycardia, or in monomorphic sustained ventricular tachycardia occurring during the electrophysiological study. The sole administration of amiodarone as medical treatment is a possibility in patients with non-sustained ventricular tachycardia and normal noninducible electrophysiological study. Amiodarone can also be given empirically to reduce the recurrence of applicable or inapplicable ICD discharges and lowers the possibility of electrical storm. However, if the patient gets too many discharges even though the pharmacological treatment is continued, radiofrequency ablation should be applied [31]. Since the electrophysiological mechanism of ventricular tachycardia in Chagas disease is re-entry, the main goal of ablation is to identify critical isthmuses that maintain the re-entrant circuit and ablate those small regions of the myocardium to prevent the exit of the electrical impulses that perpetuate the tachycardia [31]. There still remains a necessity of clear cut medicine based evidence regarding the treatment of ventricular arrhythmias in Chagas disease. Moreover, we need to be able to easily recognize those patients with CHD that are at high risk of developing abnormalities of the cardiac conduction system and malignant arrhythmias to rapidly implement specific therapies, namely, implantable cardioverter defibrillators and arrhythmia ablation procedures when needed.

\section{References}

1. Thery C, Gosselin B, Lekieffre J, Warembourg H (1977) Pathology of sinoatrial node. Correlations with electrocardiographic findings in 111 patients. Am Heart J 93: 735-740.

2. Spach MS, Dolher PC (1986) Relating extracellular potentials and their derivatives to anisotropic propagation at a microscopic level in human cardiac muscle. Circ Res 58: 356-371.

3. Centurión OA, Shimizu A, Isomoto S, Konoe A (2008) Mechanisms for the genesis of paroxysmal atrial fibrillation in the Wolff-Parkinson-White syndrome: intrinsic atrial muscle vulnerability vs. electrophysiological properties of the accessory pathway. Europace 10: 294-302.

4. Spach MS, Miller, WT, Dolber PC, Kootsey JM, Sommer JR, et al. (1982) The functional role of structural complexities in the propagation of depolarization in the atrium of the dog. Circulation Research 50: 175-191.

5. Boineau JP, Schuessler RB, Mooney CR, Miller CB, Wylds AC et al. (1980) Natural and evoked atrial flutter due to circus movement in dogs: role of abnormal atrial pathways, slow conduction, nonuniform refractory period distribution and premature beats. Am J Cardiol 45: 1167-1181.

6. Centurion OA, Isomoto S, Shimizu A, Konoe A, Kaibara M, et al. (2003) The effects of aging on atrial endocardial electrograms in patients with paroxysmal atrial fibrillation. Clin Cardiol 26: 435-438.

7. Boyden PA, Tilley LP, Albala A, Liu SK, Fenoglio JJ, et al. (1984) Mechanisms for atrial arrhythmias associated with cardiomyopathy: a study of feline hearts with primary myocardial disease. Circulation 69: 1036-1047.

8. Centurión OA, Shimizu A, Isomoto S, Konoe A, Kaibara M, et al. (2005) Influence of advancing age on fractionated right atrial endocardial electrograms. Am J Cardiol 96: 239-242.

9. Centurión OA (2015) Age-related electrophysiological changes of the atria myocardium in patients with paroxysmal atrial fibrillation. $\mathrm{J}$ Cardiol Curr Res 3: 00121.

10. Centurion OA, Isomoto S, Fukatani M, Shimizu A, Konoe A, et al. (1993) Relationship between atrial conduction defects and fractionated atrial endocardial electrograms in patients with sick sinus syndrome. Pacing Clin Electrophysiol 16: 2022-2033.

11. Pérez-Molina JA, Norman F, López-Vélez R (2012) Chagas disease in nonendemic countries: Epidemiology, clinical presentation and treatment. Curr Infect Dis Rep 14: 263-274.

12. Rassi A, Rassi A, Marin-Neto JA (2010) Chagas disease. Lancet 375: $1388-402$.

13. Stoloff Groppo A, Higushi L, Bocchi E (1987) Heart Transplantation in patients with Chagas Disease cardiomyopathy. J Heart Transplant 6: 307-312.

14. Mitelman J, Giménez L, Romero Villanueva H (2004) Y col. Electrocardiography of high resolution for the detecció of the alterations of functional mycdiculars in Chagas indeterminado. Tem de Cardiol 5: 9-12.

15. Andres DS, Irurzun IM, Defeo MM, Mola EE, Mitelman J, et al. (2008) Detecting subclinical alterations in early stages of Chagas's disease through Holter. Circulation 118: E258-E259.

16. Dias JCP (1995) Natural history of Chagas' disease. Arq Bras Cardiol 65: 359366 .

17. Bellotti G, Bocchi EA, De Moraes AV, Higuchi ML, Barbero-Marcial M, et al (1996) In vivo detection of Trypanosoma cruzi antigens in hearts of patients with chronic Chagas' heart disease. Am Heart J 131: 301-307.

18. López L, Arai K, Jiménez E, Jiménez M, Pascuzo C, et al. (2006) C-reactive protein and interleukin- 6 serum levels increase as Chagas disease progresses towards cardiac failure. Rev Esp Cardiol 59: 50-56.

19. Rossi MA (1990) Microvascular changes as a cause of chronic cardiomyopathy in Chagas' disease. Am Heart J 120: 233-236.

20. Marin-Neto JA (1998) Cardiac dysautonomia and pathogénesis of Chagas heart disease. Int J Cardiol 66: 129-131.

21. Chagas C (1916) Pathogenic processes of American trypanosomiasis. Mem Inst Oswaldo Cruz 8: 3-38.

22. Chagas C (1911) Nova entidade mórbida do homem. Resumo geral de estudos etiologic and clínico. Mem Inst Oswaldo Cruz 3: 3-59.

23. Chagas C, Villela E (1922) Cardiac form of American Trypanosomiasis. Mem Inst Oswaldo Cruz 14: 5-91.

24. Mady C, Cardoso RH, Barretto AC, da Luz PL, Bellotti G, et al. (1994) Survival 
Citation: Centurión OA, García LB (2017) Fibrosis-Induced Abnormalities of the Cardiac Conduction System and Malignant Arrhythmias in Patients with Chagas Disease. J Bioequiv Availab 9: e79. doi: 10.4172/jbb.10000e79

and predictors of survival in patients with congestive heart failure due to Chagas' cardiomyopathy. Circulation 90: 3098-3102.

25. de Paola AA, Gomes JA, Terzian AB, Miyamoto MH, Martinez Fo EE, et al. (1995) Ventricular tachycardia during exercise testing as a predictor of sudden death in patients with chronic chagasic cardiomyopathy and ventricular arrhythmias. Br Heart J 74: 293-295.

26. Rochitte CE, Oliveira PF, Andrade JM, lanni BM, Parga JR, et al. (2005) Myocardial delayed enhancement by magnetic resonance imaging in patients with Chagas' disease: a marker of disease severity. J Am Coll Cardiol 46: 15531558.

27. Laranja FS, Dias E, Nobrega G, Miranda A (1956) Chagas 9 disease. Circulation 14: $1035-1060$
28. Ribeiro AL, Cavalvanti PS, Lombardi F, Nunes Mdo C, Barros MV, et al. (2008) Prognostic value of signal-averaged electrocardiogram in Chagas disease. $J$ Cardiovasc Electrophysiol 19: 502-509.

29. Rassi A Jr, Rassi SG, Rassi A (2001) Sudden death in Chagas' disease. Arq Bras Cardiol 76: 75-96

30. Oliveira JS, Mello de Oliveira JA, Frederigue U, Lima Filho EC (1981) Apical aneurysm of Chagas' heart disease. Br Heart J 46: 432-437.

31. Sosa E, Scanavacca M, D’Avila A, Bellotti G, Pilleggi F (1999) Radiofrequency catheter ablation of ventricular tachycardia guided by nonsurgical epicardial mapping in chronic chagasic heart disease. Pacing Clin Electrophysiol 22 128-130. 